University of Windsor

Scholarship at UWindsor

\title{
Control of surface plasmon resonances in dielectrically coated proximate gold nanoparticles immobilized on a substrate
}

\author{
P. Rooney
}

A. Rezaee

S. Xu

T. Manifar

Chitra Rangan

University of Windsor

Follow this and additional works at: https://scholar.uwindsor.ca/physicspub

Part of the Physics Commons

\section{Recommended Citation}

Rooney, P.; Rezaee, A.; Xu, S.; Manifar, T.; and Rangan, Chitra. (2008). Control of surface plasmon resonances in dielectrically coated proximate gold nanoparticles immobilized on a substrate. Physical Review B - Condensed Matter and Materials Physics, 77 (23), 235446-1-235446-9.

https://scholar.uwindsor.ca/physicspub/2

This Article is brought to you for free and open access by the Department of Physics at Scholarship at UWindsor. It has been accepted for inclusion in Physics Publications by an authorized administrator of Scholarship at UWindsor. For more information, please contact scholarship@uwindsor.ca. 


\title{
Control of surface plasmon resonances in dielectrically coated proximate gold nanoparticles immobilized on a substrate
}

\author{
Patrick Rooney, ${ }^{1, *}$ Asad Rezaee, ${ }^{2}$ Songbo Xu ${ }^{2}$ Touraj Manifar, ${ }^{2}$ Abdollah Hassanzadeh, ${ }^{2}$ Ganna Podoprygorina,${ }^{3}$ \\ Volker Böhmer, ${ }^{3}$ Chitra Rangan, ${ }^{1, \dagger}$ and Silvia Mittler ${ }^{2, \ddagger}$ \\ ${ }^{1}$ Department of Physics, University of Windsor, Windsor, Ontario, Canada N9B 3P4 \\ ${ }^{2}$ Department of Physics and Astronomy, The University of Western Ontario, London, Ontario, Canada N6A $3 K 7$ \\ ${ }^{3}$ Department of Chemistry, Johannes Gutenberg University, D 55128 Mainz, Germany
}

(Received 15 March 2007; revised manuscript received 17 March 2008; published 30 June 2008)

\begin{abstract}
We present experimental and theoretical results for the changes in the optical-plasmon resonance of goldnanoparticle dimers immobilized on a surface when coated with an organic dielectric material. The plasmon band of a nanoparticle dimer shifts to a higher wavelength when the distance between neighboring particles is decreased, and a well-separated second peak appears. This phenomenon is called cross-talk. We find that an organic coating lets cross-talk start at larger separation distances than for uncoated dimers by bridging the gap between immobilized nanoparticles (creating optical clusters). We study this optical clustering effect as a function of the polarization of the applied light, of the inter-particle distance, of the surrounding environment, and of the optical properties of the coating layer. Theoretical discrete-dipole approximation calculations support the experimental absorption spectroscopy results of gold nanoparticles on glass substrates and on optical waveguides.
\end{abstract}

DOI: 10.1103/PhysRevB.77.235446 PACS number(s): 78.67.Bf, 73.20.Mf, 42.82. $-\mathrm{m}$, 41.20. $-\mathrm{q}$

\section{INTRODUCTION}

The extensive research interest in nanoscale inorganic particles has mainly been driven by their broad impact on the emerging disciplines of nanoengineering, ${ }^{1}$ nanoelectronics, ${ }^{2}$ and nanobioelectronics. ${ }^{3}$ Gold nanoparticles, in particular, have been the focus of numerous investigations in recent years because of promises offered by their optical, electronic, and chemical properties. ${ }^{4}$ Of particular interest are their tunable electronic, magnetic, and photonic properties due to quantum confinement effects, with nanocluster-based devices being envisioned as the next generation in electronics miniaturization. ${ }^{5}$

Recent years have also seen a tremendous impact on silver and gold nanoparticles and nano-objects in biological assays, detection, labeling, and sensing. The immobilization of molecules using recognition sites and the clustering of the nanoparticles into larger agglomerates with a pronounced shift in the plasmon absorption band of nanoparticles in solution are well studied. 6,7

In order to use color change during cluster formation for recognition reactions with only minute amounts of sample material available, or in a screening approach with many different recognition agents, the volume has to be minimized and the accessibility enhanced. Therefore, we use a twodimensional approach of immobilizing nanoparticles on a surface.

In this paper, we describe experimental and theoretical studies of the dependence of the optical plasmon resonance spectrum on optical cluster formation due to the coating of a dimer of nanoparticles by an organic layer. This research is challenging both for technological and theoretical reasons.

One of the technological challenges is pinning down the precise optical response of nanoparticle mini-clusters, e.g., dimers, trimers, and oligomers. ${ }^{8}$ To mimic the high sensitivity of the three-dimensional (3D) solution experiments, one must establish the particular interparticle distance at which the plasmon band both begins to shift and reaches a maximum, as well as the dependence on parameters such as the dielectric constant of the surrounding medium and the substrate. ${ }^{9-12}$ Su et al. ${ }^{13}$ did experiments and theoretical calculations for immobilized gold elliptical nanoparticles of sizes varying from 84-104 $\mathrm{nm}$. They found an exponential decay of the particle resonance redshift with increasing particle distance both theoretically and experimentally. In this paper, we investigate the optical response of hemispherical gold nanoparticles of smaller diameter (14-35 nm). At this smaller scale, we can see additional "cross-talk" peaks ${ }^{14}$ in the extinction spectrum, which are absent from the simulations with larger particles as in $\mathrm{Su}$ et al.'s work, ${ }^{13}$ and an increase in sensitivity to the particle shape. We go further and add the crucial feature of dielectric organic coatings. The presence of these coatings can extend the effective size of the metal particles and produce cross-talk features in the spectrum of the nanoparticles, whose separation would otherwise be too great to produce cross-talk.

The second challenge is the fabrication of robust and organized assemblies of these nanoscale building blocks, $,, 10,15$ where the collective structural properties and functions can be manipulated by the nature, size, form, position, and overall composition of the nanoparticles on a substrate in an easy, quick, and inexpensive fashion.

Among the various techniques developed, self-assembly was quite effective in creating ordered superlattices of nanoparticles by exploiting the molecular interactions such as van der Waals forces, chemisorptive bonding, and electrostatic interactions. ${ }^{4,5,16}$ One key structural element can be a bifunctional bridge, which links the nanoparticles to the substrate surfaces and/or allows layering of nanoparticles such as aliphatic dithiols. ${ }^{17}$ These processes can be achieved by simple beaker chemistry.

In addition, self-assembled monolayers ${ }^{18,19}$ (SAMs) have drawn special attention because their preparation is simple 

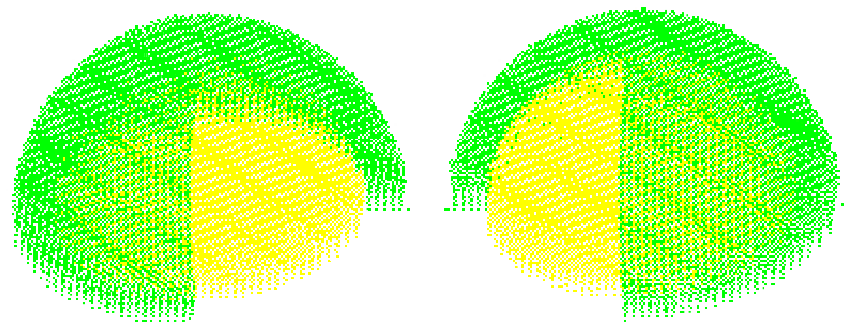

FIG. 1. (Color online) 3D picture of a discrete dipole array that models two hemispherical gold nanoparticles on a plane, coated by a dielectric material. Each point represents one dipole: the organic coating is green (dark gray) and the gold nanoparticle is yellow (light gray). The coating has been peeled back for a view of the nanoparticle beneath. The particle radius is $7 \mathrm{~nm}$, the coating thickness is $1.75 \mathrm{~nm}$, and the surface-to-surface separation (that is varied) is $1.75 \mathrm{~nm}$. The electromagnetic wave propagates along the plane of the substrate perpendicular to the interparticle axis. In the $s$-polarization, the electric field points along the interparticle axis, and in the $p$-polarization, the electric field points perpendicular to the planar substrate.

and the resultant films can be densely packed, highly ordered, and largely free of defects. In this work, SAMs of 3-mercaptopropyltrimethoxysilane (MPTS) are used as templates for the random nucleation and growth of nano-sized gold particles grown via organometallic chemical vapor deposition (OMCVD). Details on this particular method can be found elsewhere. ${ }^{17,20}$ Thiol SAMs were used to cap the nanoparticles with an organic material.

Several theoretical techniques for studying electrodynamics of nanoparticles have been developed in recent years. The Mie theory of scattering ${ }^{21}$ describes exactly the optical behavior of spherical particles of all sizes, as well as infinite cylinders. For more interesting geometries, several approximation schemes have been developed, the most successful being the finite-difference time-domain method ${ }^{22}$ and the discrete dipole approximation (DDA) ${ }^{23}$ In this paper, we use the DDA to numerically analyze the spectral behavior of dimers of immobilized gold nanoparticles. We study hemispherical particles with specific surface-to-surface separations (metal-to-metal for uncoated particles or dielectric-todielectric for coated particles), where the members of each dimer pair have flat surfaces that are coplanar. Coatings are added to the curved surface regions so that the gold-coating and coating-environment boundaries are concentric hemispheres (see Fig. 1).

In the following sections, we document our investigations of the optical plasmon resonance of surface-immobilized nanoparticles coated by an organic dielectric material. In the "Methods" section, we briefly describe the experimental methods used to prepare the nanoparticles arrays and cap the nanoparticles with an organic layer, as well as the spectroscopic methods for detection of the optical plasmon resonance. We also outline our implementation of the discrete dipole approximation method for the solution of Maxwell's equations. In the "Results and Discussion" section, we describe our experimental results and the theoretical calculations that support these results, and in "Conclusions," we note a potential application of these results.

\section{METHODS}

\section{A. Experiment}

\section{Self-assembled monolayer preparation}

Calix[4] arene heterodimer capsules were synthesized as described elsewhere. ${ }^{24}$ Dichloromethane $\left(\mathrm{CH}_{2} \mathrm{Cl}_{2}, \geq 99.8 \%\right)$ was obtained from Caledon Laboratories Ltd. Ferrocenium hexafluorophosphate $\left(\mathrm{Fc}^{+} \mathrm{PF}_{6}^{-}\right)$was obtained from SigmaAldrich. All regents and solvents were used without further purification.

SAMs of the heterodimer capsules were fabricated by formation of the heterodimer in a solution and immobilization in $10 \mu \mathrm{M}$ solutions of both dimer forming calix[4]arenes in dichloromethane for no less than $16 \mathrm{~h}$. SAM formation of ferrocenium-filled capsules were performed in a solution with $0.1 \mathrm{mM}$ of the heterodimer capsules with ferrocenium (ferrocenium hexafluorophosphate was $20 \%$ over plus) in dichloromethane for $25 \mathrm{~h}^{24}$

For OMCVD, gold-nanoparticle-fabrication templateSAMs of MPTS (Fluka, 97.0\%, used without further purification) are necessary. Before the silanization process, glass substrates (Schott BGG 11) were first cleaned by 5-min ultra sonication in acetone, rinsing with Milli-Q-water (Milli-Q, $\rho>18 \mathrm{M} \Omega \mathrm{cm}$, Millipore), 5-min ultra sonication, and rinsing with water. Glass substrates were immersed in piranha solution (70 vol \% $\mathrm{H}_{2} \mathrm{SO}_{4}+30$ vol $\% \mathrm{H}_{2} \mathrm{O}_{2}$ ) for $30 \mathrm{~min}$ at 90- $100{ }^{\circ} \mathrm{C}$, then rinsed thoroughly with Milli-Q-water and dried with $\mathrm{N}_{2}$. Contact angle measurement confirmed the hydrophilic nature of the slides $\left(\theta<2^{\circ}\right) .1 \%$ solution of MPTS in dry ethanol was freshly prepared under nitrogen atmosphere (glove box) and used quickly. The cleaned substrates were immersed for $2 \mathrm{~h}$ at room temperature in a sealed container. They were rinsed with ethanol, dried with N2, and subsequently placed in a vacuumed oven at $94{ }^{\circ} \mathrm{C}$ for $2 \mathrm{~h}$ (60 min heating +60 min cooling down under vacuum). The freshly prepared mercaptosilane SAMs were used immediately as substrates for the OMCVD gold nanoparticles. This immobilization reaction can lead to monolayer formation of MPTS. Nevertheless, often this reaction leads to larger film thickness due to a polymerization reaction triggered by enhanced water content during the immobilization reaction. The theoretical thickness of the monolayer is $0.77 \mathrm{~nm} \mathrm{ac}$ cording to the length of the molecule. With ellipsometry, the monolayer thickness has been determined as $0.80 .1 \mathrm{~nm}$ with a roughness of $0.14 \mathrm{~nm}^{25}$ Our atomic force microscopy (AFM) measurements show a thickness of $6 \mathrm{~nm}$ with a roughness of $0.16 \mathrm{~nm}$.

To cap SAMs, 1-dodecanethiol (Sigma-Aldrich, product of ATOFINA Chemicals, Inc., 98+\% purity) was used without further purification and dissolved in ethanol (Commercial Alcohols Inc., Brampton, ON, puriss.) at a concentration of 1 $\mathrm{mM}$. The SAM formation was carried out for $15 \mathrm{~h}$. The samples were then cleaned by rinsing extensively with ethanol and Milli-Q-water (Milli-Q, $\rho>18 \mathrm{M} \Omega \mathrm{cm}$, Millipore) and dried with $\mathrm{N}_{2}$.

\section{Organometallic chemical vapor deposition of gold onto self- assembled monolayers}

The vapor deposition of $\left[\left(\mathrm{CH}_{3}\right)_{3} \mathrm{P}\right] \mathrm{AuCH}_{3}{ }^{26}$ onto SAMs was carried out in a home-built, vacuum-sealed, glass reactor 
chamber, which contained the SAM sample and a small glass vessel with $20 \mathrm{mg}$ of the gold precursor. The reactor was evacuated $\left(p_{\max }=2.0 \times 10^{-2} \mathrm{hPa}\right)$ and placed in an oven at $63{ }^{\circ} \mathrm{C} .{ }^{20}$ The deposition time was varied from 15 to $120 \mathrm{~min}$. Subsequently, the SAMs were quickly removed, rinsed with dry ethanol, and stored under inert conditions. Hemispherical gold nanoparticles with a range of average nanoparticle diameters (from 14 through $40 \mathrm{~nm}$ ) were produced and characterized by AFM and electron microscopy. The average distance between these statistically grown nanoparticles for the case of 30-min growth time is in the order of $60 \mathrm{~nm}$ but has a very wide distribution. We find distances as small as a few $\mathrm{nm}$ (short distance tail of distribution) but also distances as large as a few $100 \mathrm{~s}$ of $\mathrm{nm}$ (long distance tail of distribution). The average distances decrease with increasing growth time and vice versa.

\section{3. $U V$-visible spectroscopy}

UV-visible absorption spectra were obtained using a Lambda 850 UV-visible recording spectrophotometer (PerkinElmer). Baseline correction was performed before scanning the samples. SAM films were scanned with clean glass as its reference. For OMCVD Au samples, SAM films were used as its reference.

\section{Evanescent waveguide absorption spectroscopy}

Schott BG 11 glass samples were used to fabricate ionexchanged slab waveguides in a $100 \% \mathrm{AgNO}_{3}$ melt at $225^{\circ} \mathrm{C}$ in a tube furnace (YOKOGAWA, model STF55346C-1) for 4 min. ${ }^{27}$ This leads to waveguides ${ }^{28}$ with one mode in both polarization directions. The end fires were polished. Light from a white light source with an emission spectrum from 360 to $2000 \mathrm{~nm}$ (Ocean Optics, Dunedin, Florida, USA, HL-2000-HP with $20 \mathrm{~W}$ output) was fed into the waveguide via the end fire coupling with a glass fiber and a microscope objective (Newport, $20 \mathrm{x}$ ). The light propagates in the channel waveguide as a waveguide mode in transverse electric (TE) or transverse magnetic (TM) polarization (chosen by a polarizer in front of the coupling objective), exhibiting an evanescent field on its free accessible surface with respective polarization. The transmitted light is collected via a second microscope objective (Newport, $20 \mathrm{x}$ ) and fed via a second glass fiber into a spectrometer (Ocean Optics, Dunedin, Florida, USA, HR-2000 spectrometer). The absorption spectra of the gold nanoparticles recorded this way are normalized by the spectrum of the silanized waveguide.

\section{B. Theory}

We solve Maxwell's equations for the scattering of white light (400-800 nm) from gold nanoparticle dimers covered by a dielectric material. There are several methods available: the modified long-wavelength approximation ${ }^{29}$ and the single-dipole approximation ${ }^{14}$ are two approximate methods. Another popular method is the finite-difference time-domain (FDTD) approach. ${ }^{22,30} \mathrm{We}$ also note several theoretical results presented in the excellent book by Bedeaux and Vlieger. ${ }^{31}$ In this work, we use the well-known discrete di- pole approximation (DDA) developed by Draine and Flatau. ${ }^{32}$

The DDA relies on the simple but powerful approximation of a continuous material by a discretized target of $N$ point dipoles. In principle, arbitrary discretizations are allowed; however, the fast Fourier transform techniques in the program DDSCAT ${ }^{32}$ (version 6.0$)^{2}$ require cubic arrays. Draine and Flatau have noted that one of the limitations of the method is the faithful representation of target surfaces. This problem could be circumvented by increasing dipole density in high-curvature surface regions, but this solution is impossible for cubic arrays. Fortunately, our hemispherical particles have a low surface-to-volume ratio, and this limitation is not a concern.

Each dipole is uniquely described by its grid location $r_{i}$ and polarizability $\alpha_{i}$. The polarizabilities are calculated from the complex dielectric function $\varepsilon_{i}$ of the material using the Clausius-Mossotti relation, ${ }^{23}$

$$
\frac{\varepsilon_{i}-1}{\varepsilon_{i}+2}=\frac{n_{d} \alpha_{i}}{3},
$$

where $n_{d}$ is the number density of the array. The dielectric function of gold in the optical range was taken from Palik. ${ }^{33}$ For calculations of targets in nonvacuum surroundings, the dielectric function must be adjusted according to that of the surrounding medium. ${ }^{32}$ In this article, we will repeat our vacuum simulations in water $(n=1.33)$.

The polarizabilities give a relation between the polarizations of the dipoles and the local electric field (vectors are represented by boldface),

$$
\vec{P}_{i}=\alpha_{i} \vec{E}_{\mathrm{loc}, i}\left(\vec{r}_{i}\right) .
$$

The local electric field has two contributions: the incident field and the fields of all other dipoles,

$$
E_{\mathrm{loc}, i}\left(\vec{r}_{i}\right)=E_{0} \exp \left(i \vec{k} \cdot \vec{r}_{i}\right)-\Sigma_{j} A_{i j} \cdot P_{j},
$$

where $A_{i j}$ is an interaction matrix that depends on pairwise separations $r_{i j}$ and the magnitude of the wave number $k$. Note that the incident light is assumed to be a plane wave, a constraint demanded by the program DDSCAT. In the experiment, the nanoparticles are illuminated by the evanescent field from the waveguide substrate, but the survival depth of the intensity is of the order of a few hundred nanometers, which dwarfs the scale of our particles so that the planewave approximation is a good one.

From the above equations, one may construct a system of three $\mathrm{N}$ complex, linear equations,

$$
\Sigma_{j} A_{i j}^{\prime} \cdot P_{j}=E_{0} \exp \left(i \vec{k} \cdot \vec{r}_{i}\right)
$$

After solving for the polarization $P_{j}$, one may use it to construct the near-field and far-field optical properties of the target. We are interested in the extinction cross section of the particles or the sum of the absorption and scattering cross sections. In our study, the absorption was typically found to overwhelm scattering by an order of magnitude.

The validity criterion for the DDA is the long-wavelength limit: $|m| k d<1$, where $m$ is the complex refractive index, $k$ is the wave number, and $d$ is the grid spacing. We choose the 
grid spacing to be small enough so as to satisfy this criterion. We compared the DDA calculations for a coated gold sphere (both in air and in an aqueous medium) with that from Mie theory, ${ }^{21}$ and find excellent agreement.

In this work, we ignore the effects of the substrate. That is, the substrate's refractive index is taken to be the same as that of the ambient medium. From investigations on substrate effects by Schatz and co-workers, ${ }^{34}$ it is clear that substrate effects become significant when there is direct contact between the nanoparticle and the substrate and depend on the fraction of surface area of the nanoparticle in contact. Since in the experiments, the nanoparticles are not in direct contact with the waveguide surface but are immobilized via silane chemistry, it is reasonable to ignore the substrate in these calculations.

A practical limitation is memory space which limited us to an array of $135 \times 135 \times 135$ dipoles. Because of this and the validity criterion, we calculate all of the results for 14 nm-diameter sized particles, and all reported results are for dimers. Trimers and tetramers were also studied with coarser discretization, and they showed very similar behaviors to the dimers.

A picture of a typical target is seen in Fig. 1. The convergence of numerical solutions indicates that this arrangement appears to adequately approximate the continuous target. Two fully coated and separated nanoparticles are represented by 67228 discrete dipoles. The grid spacing is set to 0.35 $\mathrm{nm}$, giving bare particles with a physical diameter of $14 \mathrm{~nm}$ a height of $7 \mathrm{~nm}$ and a coating of thickness $1.75 \mathrm{~nm}$, values which are typical for the smallest structures that have been produced and used in this experiment. Incident light propagates transverse to the interparticle axis and parallel to the substrate surface. Extinction spectra are calculated for both $s$-polarization (with electric field vector parallel to the interparticle axis) and $p$-polarization (perpendicular to the waveguide plane). Our results show a strong dependence on the polarization of the incident field.

\section{RESULTS AND DISCUSSION}

First, we study the optical plasmon resonance of bare nanoparticles as a function of interparticle spacing. This effect was studied experimentally on immobilized OMCVD gold nanoparticles with evanescent waveguide absorption spectroscopy. Figure 2(a) depicts the evanescent waveguide absorption (EWA) spectra for a 40 min-grown sample of gold nanoparticles without any coating in air. Each curve shows a different polarization of the electric field. An increased absorbance between 700 and $800 \mathrm{~nm}$ is found in the in-plane $s$-polarization but not in the out-of-plane $p$-polarization. Theoretical work by Jensen et al. ${ }^{14}$ showed that when two metal nanoparticles approach each other, cross-talk causes the plasmon peak to shift to longer wavelengths and the appearance of an additional peak, and these are referred to as the dipolar and quadrupolar peaks, respectively. This effect can be explained by the onset of a crosstalk behavior between neighboring particles in these random arrays of nanoparticles, i.e., the nanoparticles are close enough such that the evanescent fields between the two over-

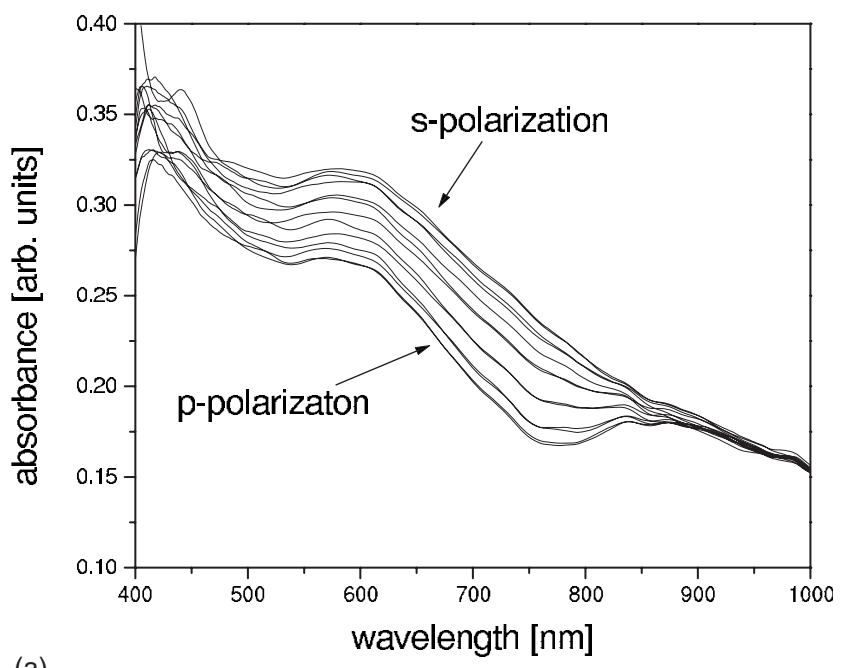

(a)

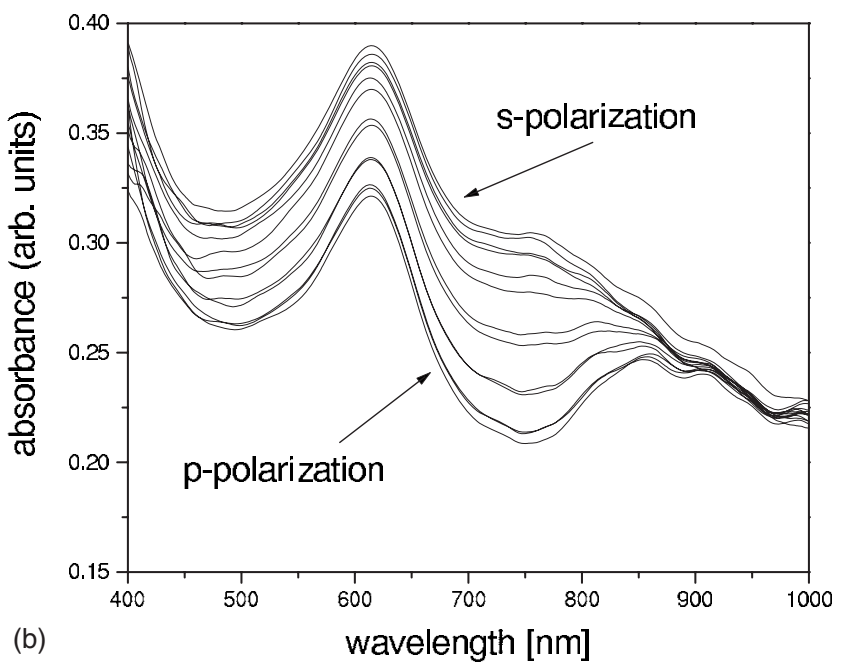

FIG. 2. Measured EWA spectra of 40 min-grown nanoparticles of first generation with successively changed polarization in $15^{\circ}$ steps: $0^{\circ}$ and $180^{\circ}$ represent a pure TE ( $s$-polarized) mode whereas $90^{\circ}$ represents a pure TM ( $p$-polarized) mode. (a) without coating and (b) with a 1,8 octanedithiol SAM.

lap. In Fig. 2(a), one can identify a peak at $600 \mathrm{~nm}$ as the dipolar peak of the individual nanoparticles and an emerging shoulder between 650 and $800 \mathrm{~nm}$ due to an onset of crosstalk between neighboring nanoparticles in $s$-polarization.

Note that since the OMCVD-grown nanoparticles are not equally spaced, cross-talk starts between some pairs of nanoparticles that are close enough, but the other nanoparticles still show a single particle response. Thus, the "cross-talk" peak is dwarfed by the single particle dipolar peak. As expected, the cross-talk peak appears only in the in-plane polarization. We note that similar features observed in the extinction spectra of metal nanoparticle arrays have been explained by propagating surface plasmon modes, ${ }^{12}$ increase in size effects, ${ }^{35}$ or bound states. ${ }^{36}$ These, however, do not explain the experimental results described below.

In Fig. 2(b), the same nanoparticles have been coated with a SAM of 1,8 octanedithiol $(n=1.45$ and a film thickness of $\approx 7.5 \AA$ ). We find an enhanced cross-talk behavior for the 


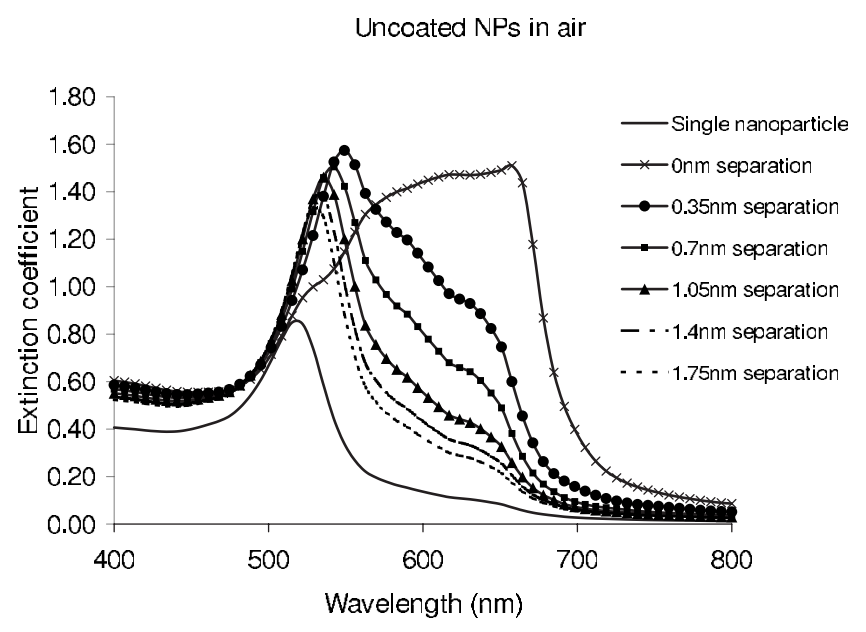

Dielectric coated NPs in air

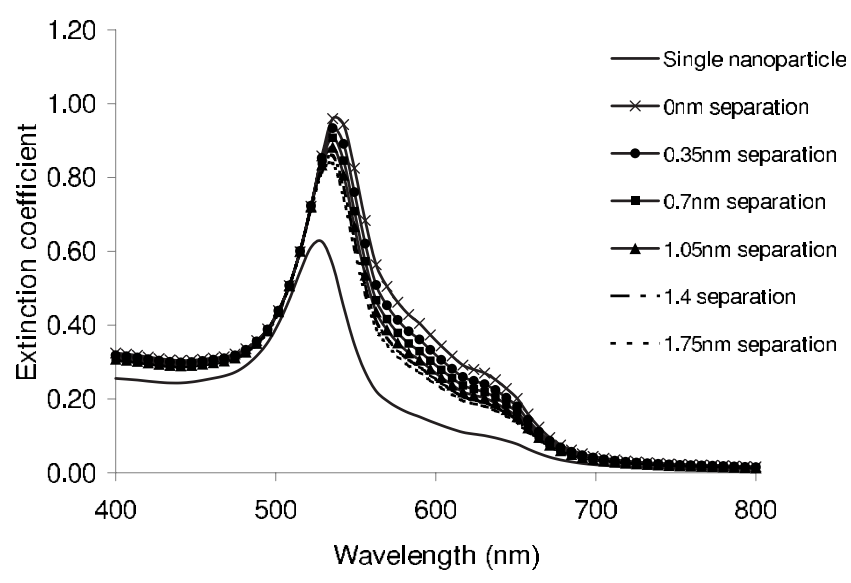

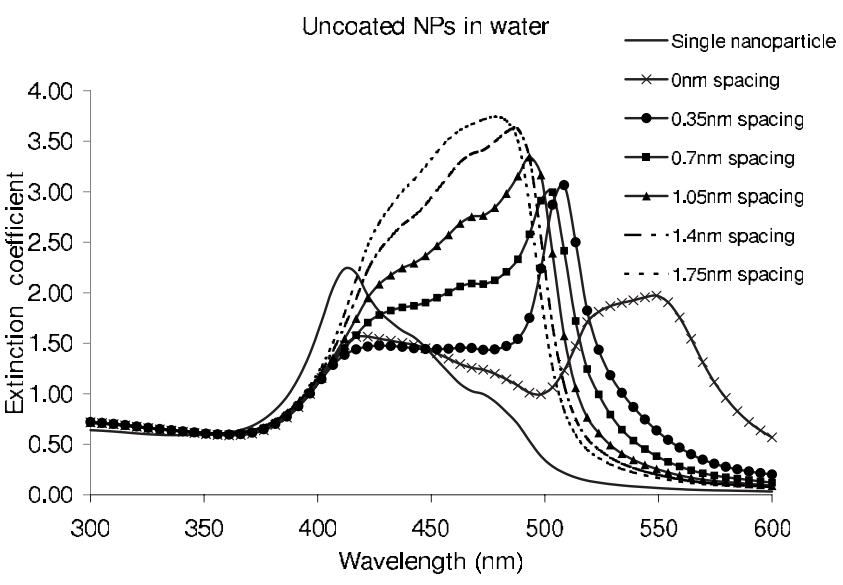

Dielectric coatec NPs in water

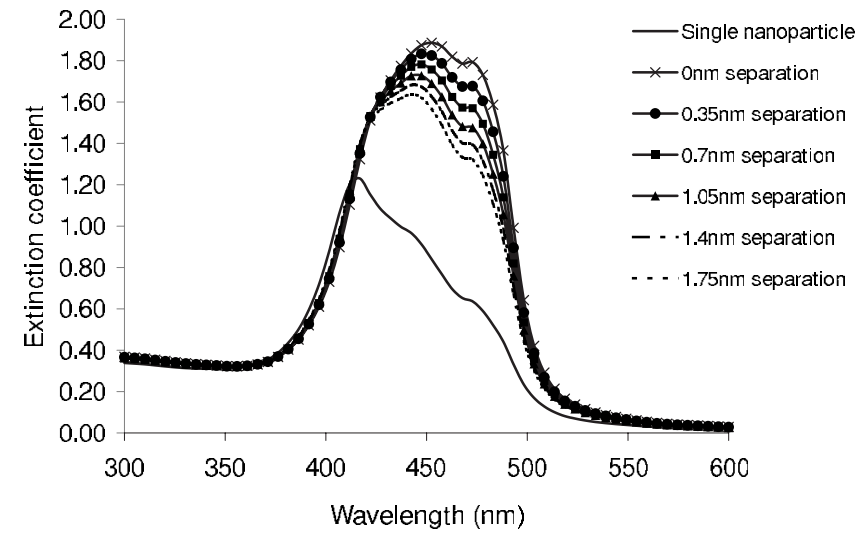

FIG. 3. Calculated extinction spectra for two hemispherical gold nanoparticles (NPs) with varying interparticle separations. Particles are (top left) uncoated in air, (top right) coated in air, (bottom left) uncoated in water and (bottom right) coated in water. All coatings have a refractive index of 1.45. Particle radius is $7 \mathrm{~nm}$ and coating thickness is $1.75 \mathrm{~nm}$. Separation is measured as distance between surfaces, which for coated particles means the coating-to-coating distance.

capped nanoparticles with a pronounced cross-talk peak in $s$-polarization at $755 \mathrm{~nm}$. The dipolar peak shift in both the polarization directions can be attributed to the increase in the ambient refractive index and an increase in the size of the nanoparticle. The absence of the second peak in the out of plane $p$-polarization indicates that size effects alone ${ }^{35}$ cannot explain the spectrum. Also, since this effect is observed by the addition of a dielectric coating, the propagating surface plasmon mode explanation ${ }^{12}$ does not seem feasible. The enhancement of the dipolar cross-talk peak in only the in-plane polarization spectra suggests that the decrease in distance between the surfaces of the nanoparticles due to the coating promotes the cross-talk. Clearly, cross-talk is enhanced by the addition of the organic coating when the distance between the nanoparticle centers stays the same. We call this effect "optical clustering" because even though the physical particles do not overlap, the spectra show signatures of the nanoparticle clustering.

The experiments were repeated for samples with average nanoparticle diameters between 14 and $40 \mathrm{~nm}$, and the spectra showed the same behavior. Theoretical calculations performed using the DDA support these experimental results.
For calculations, the nanoparticles are chosen to be $7 \mathrm{~nm}$ in radius (corresponding to the sample with the smallest average size in the experiments). Based on experimental estimates, we fix the refractive index of the coating to $n=1.45$ and the coating thickness to $1.75 \mathrm{~nm}$. The incident light propagates along the surface perpendicular to the interparticle axis.

The most interesting results are obtained when the electric field is $s$-polarized, i.e., the electric field vector is along the interparticle axis. We start with particles that are minimally separated, i.e., touching, and slowly separate them. Figure 3, top left, shows results for these separations for uncoated particles in air. Beginning with the irregular spectrum for the zero-separation case, we see a gradual relaxation back toward the single-particle spectrum for widely separated particles. The cross-talk feature is clear when the nanoparticles are close together. These results agree qualitatively with the calculations performed by Jensen et al., ${ }^{14}$ who have seen resonance shifts in oblate spheroidal silver nanoparticles with decreasing interparticle distance.

When the nanoparticles are coated with an organic layer, two effects are evident as seen in Fig. 3, top right. Compar- 


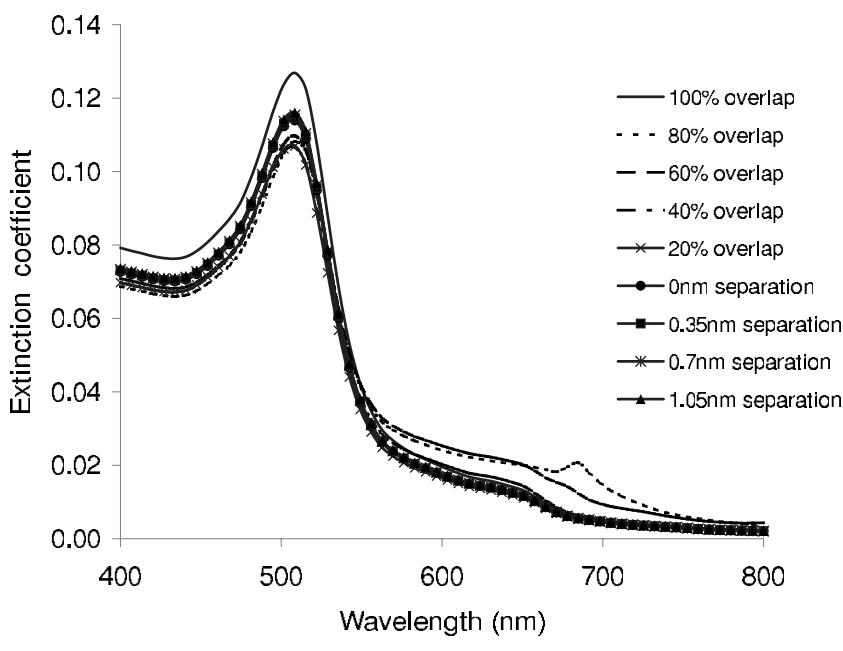

FIG. 4. Calculated extinction spectra for target illuminated with $p$-polarized light (the light polarization is perpendicular to the plane on which the NPs are immobilized). Both overlapped and separated targets are shown. Simulations are done in air.

ing with the single nanoparticle spectra, one can see the shift in the spectrum due to the increase in the size of the nanoparticles upon addition of the coating, and this is fairly small. However, even when the nanoparticles' coatings are not touching (i.e., the metal surfaces themselves are well separated), there is a shift of the single particle dipolar plasmon resonance and a pronounced cross-talk feature. Note that the position of this latter peak does not change with change in the distance between the coating surfaces. Therefore, we do not attribute this peak to the formation of bound states between the two nanoparticles. ${ }^{36}$

The bottom left and right spectra of Fig. 3 show the calculated plasmon resonance shifts for bare and coated nanoparticles, respectively, as a function of interparticle separation in an aqueous medium. The single nanoparticle spectra (both bare and coated) in a water medium are markedly different from those in air. Along with the redshift expected due to an ambient medium, additional structure is seen even for an uncoated single nanoparticle in water. Recall that our nanoparticles are hemispherical in shape. This structure is not seen for a single spherical nanoparticle in water (both in DDA and Mie theory calculations); therefore, we conclude that the presence of an aqueous medium augments the sensitivity of the spectrum to the shape of the nanoparticles. Also, the cross-talk shifts are evident even for large interparticle separations. Thus, the presence of an aqueous medium enhances the cross-talk. We observe that for both dry and wet environments, the relaxation from the zero separation behavior to the widely separated (or single nanoparticles) behavior is roughly a function of the distance between outer surfaces even if the outer surface is not that of the gold nanoparticle.

Next, we study the effect of polarization of the incident light on the shift of the optical plasmon resonance. When the incident light is $p$-polarized, the plasmon resonance shift is not as pronounced as shown in the $s$-polarized case, as seen in Fig. 4. Coating the nanoparticles increases the effective size of the nanoparticles. The $s$-polarized light also acts as a probe of the overlapping electromagnetic fields between the nanoparticles, i.e., of the optical clustering effect. The $p$-polarized light is only a probe of increased nanoparticle size but not of the overlap of the evanescent fields and, therefore, does not experience as pronounced a spectral shift. This effect is evident precisely because we have immobilized nanoparticles; for nanoparticles in solution, this effect is not seen because the shift is averaged over all orientations.? Therefore, for the rest of this paper, we present results only with $s$-polarized light.

In Fig. 5, we show the effect of increasing the refractive index of coatings in an ambient medium of air. We have chosen calix[4]arene heterodimer capsules as a material for a thin film coating. These capsules can be filled with various guests, and when self-assembled into a monolayer, form a uniform organic layer of thickness $\sim 50 \pm 2 \AA$ around the nanoparticle but with an adjustable refractive index, depending on the guest molecule located in the cavity. These calix[4]arene capsules carry a sulfur moiety to allow the binding as a self-assembled monolayer to the gold nanoparticles. The SAM of the dichloromethane-filled capsules has a refractive index $n=1.480$ and the ferrocenium-ion-filled capsule SAM a refractive index $n=1.466 .{ }^{24} \mathrm{UV}$-visible transmission absorption spectra in air and water of the bare nanoparticles fabricated by OMCVD (30-min deposition time) and particles capped with a SAM made out of dichloremethanefilled and ferrocenium-filled capsules are shown in Fig. 3.

In Fig. 5, a plasmon band around $536 \mathrm{~nm}$ is found for the bare gold nanoparticles in air. The spectra for the capped nanoparticles are redshifted by $\Delta \lambda=17$ to $553 \mathrm{~nm}$ in the dichloromethane case $(n=1.48)$ and by $\Delta \lambda=7$ to $543 \mathrm{~nm}$ in the ferrocenium-ion-filled case $(n=1.466)$ in air. This agrees well with the theory: the larger the refractive index of the cap, the larger is the plasmon resonance shift for a given coating thickness. This observation is true in water as well. The dichloromethane-filled capsules show a shift to $261 \mathrm{~nm}$ and the ferrocenium-filled capsules a shift to $551 \mathrm{~nm}$. Again,

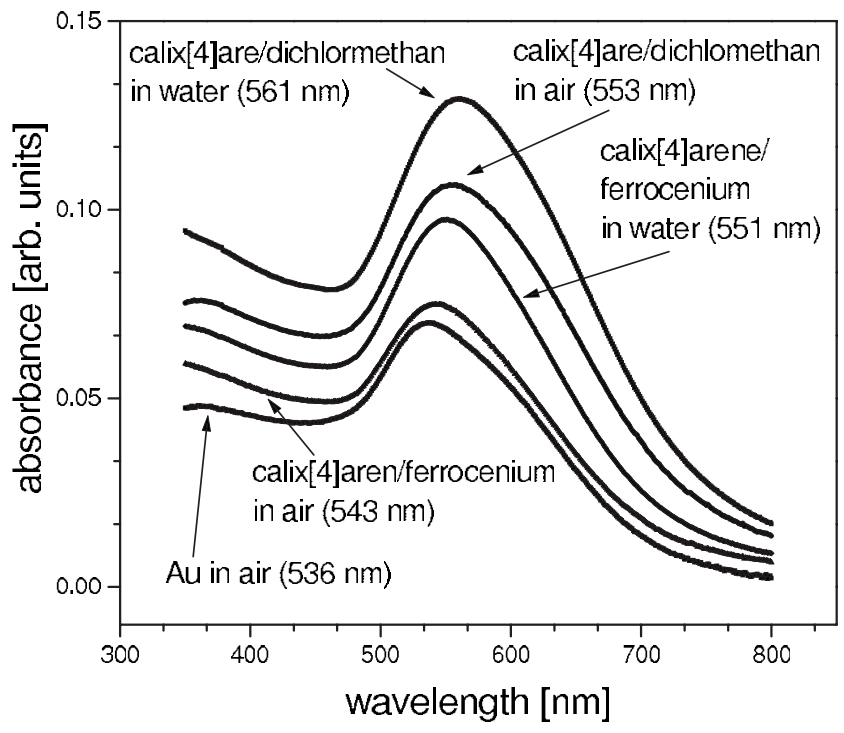

FIG. 5. Measured UV-visible spectrum of OMCVD gold nanoparticles (25 min-OMCVD grown) bare and with self-assembled monolayers of calix[4]arene heterodimers capsules filled with ferrocenium or dichloromethane in air and water. 


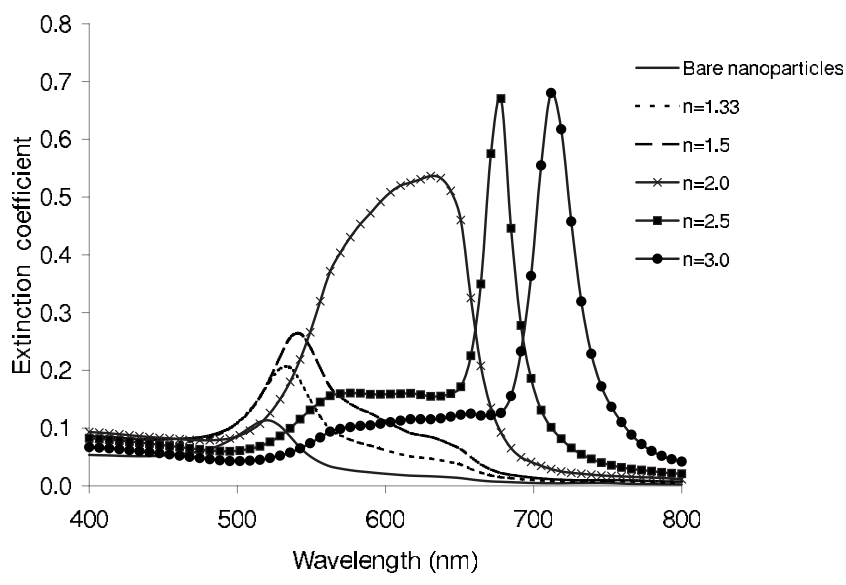

FIG. 6. Calculated extinction spectra for nanoparticles with various coating refractive indices. Particle radius is $7 \mathrm{~nm}$ and coating thickness is $7 \mathrm{~nm}$. Surface-to-surface separation is $14 \mathrm{~nm}$ for bare nanoparticles and $0 \mathrm{~nm}$ for coated particles. Calculations are shown for surrounding media of air; those for water are published elsewhere. $^{24}$

here, a difference of $10 \mathrm{~nm}$ between the two coatings is found.

This result agrees qualitatively with calculations. In Fig. 6 , we show calculations for coatings of increasing refractive index in air. For this calculation only, we have exaggerated the coating thickness to equal the particle radius $(7 \mathrm{~nm})$ in order to see the effect more clearly. Therefore, the gold-togold separation is a full $14 \mathrm{~nm}$, while there is no coating-tocoating separation. There is a small shift of the resonance for coatings with typical refractive indices $(n=1.33,1.5)$. For coatings with higher (unphysical) indices, there is a strong redshift of the resonance peak.

Figure 7 depicts UV-visible transmission spectra in air and water of gold nanoparticles grown for $35 \mathrm{~min}$ with and without a cap of 1-dodecanthiol $(n=1.5$ and film thickness $\sim 11 \AA$ ). The bare nanoparticles in air show a peak maxi-

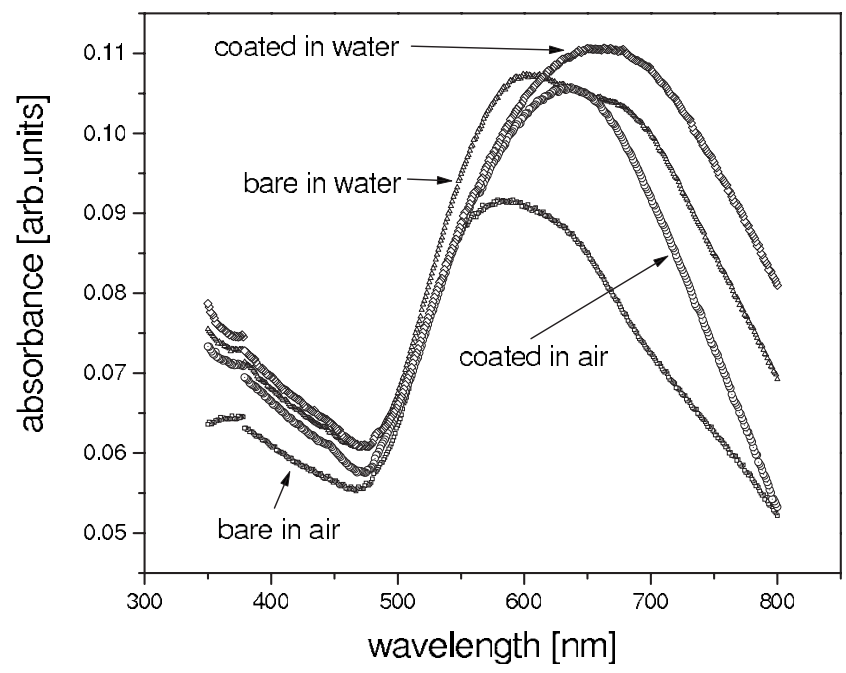

FIG. 7. Measured UV-visible spectrum of OMCVD gold nanoparticles (35 min OMCVD grown) bare and with self-assembled monolayers of 1-dodecanthiol in air and water.
Overlapping NPs in air
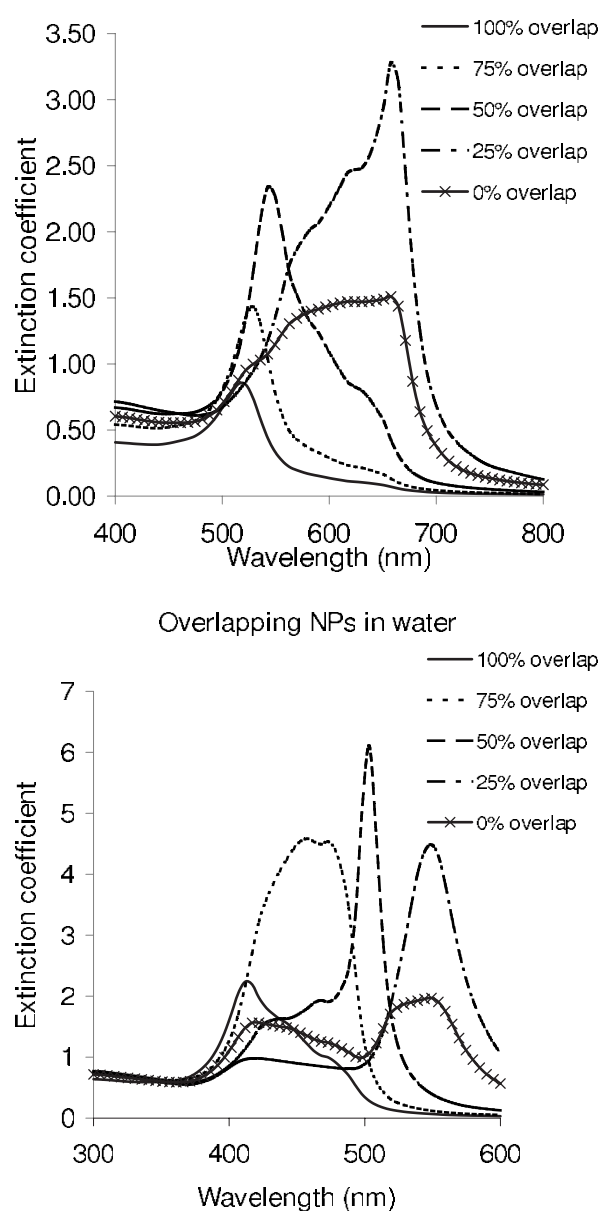

FIG. 8. Calculated extinction spectra for overlapping particles. The overlap factor is given as a percentage ratio of the center-tocenter distance to the particle diameter. Particles are aligned transverse to light propagation. Particle radius is $7 \mathrm{~nm}$ and particles are uncoated. Surrounding medium is (left) air and (right) water.

mum at $580 \mathrm{~nm}$ with a shoulder around 600-700 nm. In water, this broad peak is shifted slightly to $610 \mathrm{~nm}$ but depicts now a clear second feature around $675 \mathrm{~nm}$. As already mentioned, these OMCVD particles grow with unequal sizes and unequal interparticle spacings, and here, we observe again the onset of first interparticle interactions due to crosstalk already for the bare particles even when the single particle contributions to the absorption spectra are still a dominant feature. In the coated cases, one can observe broad peaks without internal structure; however, the peak shifts to $635 \mathrm{~nm}$ in air and $665 \mathrm{~nm}$, which means an increase to $\Delta \lambda$ $=55 \mathrm{~nm}$ in comparison to the $7-17 \mathrm{~nm}$ shifts in the calix[4]arene case.

To show how the shifts due to coatings are an extension of the shape-dependence of bare particles, we performed calculations for bare hemispherical particles that overlap, essentially representing a distorted single particle. In Fig. 8, left, we see the dependence of the resonance on overlap. When the overlap is $100 \%$, so that the particles completely coincide, we can see only a single resonance peak. As the particles separate, this peak shifts to the infrared, while a second 
peak emerges and becomes quite pronounced for $25 \%$ overlap. At $0 \%$ overlap (just touching), the particles have a very irregular spectrum. This is an example of the known effect that nanoparticles, immobilized or not, have plasmon bands that are sensitive to particle shape. ${ }^{14}$ In Fig. 8, right, the simulation is repeated for particles in an aqueous environment. The behavior is similar as before although here, the quadrupolar feature is visible even in the $100 \%$ overlap case, and the dipole shift is even more pronounced by a good 100 $\mathrm{nm}$. Clearly, shape dependence is enhanced in water. Both of these results are obtained in the $s$-polarized case-results were wholly uninteresting for the $p$-polarized case. This is intuitively satisfying: A single particle is effectively being elongated in the $s$-direction, while no enlargement occurs in the $p$-direction.

\section{CONCLUSIONS}

We have studied theoretically and experimentally the optical response of coated gold nanoparticles and analyzed the influences of the spacing between them, of the optical properties of the coatings, and of the surrounding materials. We find that cross-talk can be induced in moderately separated nanoparticles by coating them with an organic layer. This cross-talk can be detected only in the in-plane s-polarization direction of the incident light. Organic coatings with a fixed thickness but increasing refractive index lead to a shift of the plasmon resonance toward longer wavelengths and enhance a broad spectral feature interpreted as the "cross-talk" contribution in the calculations. The addition of an aqueous medium augments these features.

The role of facilitator that the coating plays, together with the fact that the binding of this coating is specific to a particular organic species, suggests a potential sensing application. Because the gold nanoparticles are immobilized, spatial separations should be controlled so that pronounced shifts are seen for coated particles only. Since these results are enhanced in an aqueous medium, small amounts of organic solutes may be detectable by observing the spectral location of the resonance peaks.

\section{ACKNOWLEDGMENTS}

The authors would like to thank NSERC, the Ontario Photonics Consortium [through Ontario Research and Development Challenge Fund (ORDCF)], CFI, ORF, and OIF, Ontario Centres of Excellence (MMO Project No. SC60134 and CMM AuTek Project No. BM60148), the Canadian CRC program as well as the Deutsche Forschungsgemeinschaft (Grant No. SFB 625) for financial support. Calculations were carried out on the SharcNet supercomputing network of Canada.
*Present address: Department of Mathematics, The University of Michigan, Ann Arbor, MI 48104.

†Corresponding author; theory: rangan@uwindsor.ca

†Corresponding author; experiments: smittler@uwo.ca

${ }^{1}$ Nanosystems, Molecular Machinery, Manufacturing and Computation, edited by K. E. Drexler (Wiley, New York, 1992); F. L. Carter, A. Schultz, and D. Duckworth, in Molecular Electronic Devices, edited by F. L. Carter (Decker, New York, 1987), p. 183.

${ }^{2}$ P. Ball and L. Garwin, Nature (London) 355, 761 (1992); Molecular Electronics, edited by G. J. Ashwell (Wiley, New York, 1992).

${ }^{3}$ I. Willner, E. Katz, and B. Willner, in Sensors Update, edited by H. Baltes, W. Göpel, and J. Hesse (Wiley, Weinheim, 1999), Vol. 5, p. 45; W. Göpel, Biosens. Bioelectron. 13, 723 (1998).

${ }^{4}$ X. Y. Zhang, L. D. Zhang, Y. Lei, L. X. Zhao, and Y. Q. Mao, J. Mater. Chem. 11, 1732 (2001); S. Sun, C. B. Murray, D. Weller, L. Folks, and A. Moser, Science 287, 1989 (2000); C. J. Kiely, J. Fink, M. Brust, D. Bethell, and D. J. Schiffrin, Nature (London) 396, 444 (1998); D. I. Gittins, D. Bethell, D. J. Schiffrin, and R. J. Nichols, ibid. 408, 67 (2000); R. G. Osifchin, R. P. Andres, J. I. Henderson, C. P. Kubiak, and R. N. Dominey, Nanotechnology 7, 412 (1996); Colloidal Gold: Principles, Methods and Applications, edited by M. A. Hayat (Academic, San Diego, 1989); U. Kreibig and M. Vollmer, Optical Properties of Metal Clusters (Springer-Verlag, Berlin, 1995); A. A. Mikhailovsky, M. A. Petruska, Kuiru Li, M. I. Stockman, and V. I. Klimov, Phys. Rev. B 69, 085401 (2004); K. Lindfors, T. Kalkbrenner, P. Stoller, and V. Sandoghdar, Phys. Rev. Lett. 93,
037401 (2004); L. A. Sweatlock, S. A. Maier, H. A. Atwater, J. J. Penninkhof, and A. Polman, Phys. Rev. B 71, 235408 (2005); A. Hohenau, J. R. Krenn, J. Beermann, S. I. Bozhevolnyi, S. G. Rodrigo, L. Martin-Moreno, and F. Garcia-Vidal, ibid. 73, 155404 (2006).

${ }^{5}$ A. P. Alivisatos, Science 271, 933 (1996); A. P. Alivisatos, J. Phys. Chem. 100, 13226 (1996); D. L. Feldheim and C. D. Keating, Chem. Soc. Rev. 27, 1 (1998).

${ }^{6}$ C. Mirkin, R. L. Letsinger, R. C. Mucic, and J. J. Storhoff, Nature (London) 382, 607 (1996); K. Aslan, J. Zhang, J. R. Lakowicz, and C. D. Geddes, J. Fluoresc. 14, 391 (2004); M. D. Malinsky, K. L. Kelly, G. C. Schatz, and R. P. Van Duyne, J. Am. Chem. Soc. 123, 1471 (2002); T. Ambjörnsson, G. Mukhopadhyay, S. P. Apell, and M. Käll, Phys. Rev. B 73, 085412 (2006).

${ }^{7}$ A. J. Haes, D. A. Stuart, S. Nie, and R. P. van Duyne, J. Fluoresc. 14, 355 (2004).

${ }^{8}$ M. Quinten and U. Kreibig, Appl. Opt. 32, 6173 (1993).

${ }^{9}$ C. Sönnichsen, S. Geier, N. E. Hecker, G. von Plessen, J. Feldmann, H. Ditlbacher, B. Lamprecht, J. R. Krenn, F. R. Aussenegg, V. Z.-H. Chan, J. P. Spatz, and M. Möller, Appl. Phys. Lett. 77, 2949 (2000).

${ }^{10}$ G.-J. Zhang, R. Möller, R. Kreyschmer, A. Csaki, and W. Fritsche, J. Fluoresc. 14, 369 (2004).

${ }^{11}$ A. Pinchuk, SPIE-Nanotechnology E-Newsletter, March 2005 (unpublished); J. J. Mock, D. R. Smith, and S. Schultz, Nano Lett. 3, 385 (2003); A. Pinchuk, A. Hilger, G. von Plessen, and U. Kreibig, Nanotechnology 15, 1890 (2004).

${ }^{12}$ N. Félidj, J. Aubard, G. Lévi, J. R. Krenn, G. Schider, A. Leitner, 
and F. R. Aussenegg, Phys. Rev. B 66, 245407 (2002).

${ }^{13}$ K.-H. Su, Q.-H. Wei, X. Zhang, J. J. Mock, D. R. Smith, and S. Schultz, Nano Lett. 3, 1087 (2003).

${ }^{14}$ T. Jensen, L. Kelly, A. Lazarides, and G. C. Schatz, J. Cluster Sci. 10, 295 (1999).

${ }^{15}$ E. Hao, G. C. Schatz, and J. T. Hupp, J. Fluoresc. 14, 331 (2004).

${ }^{16}$ S. Chen, J. Phys. Chem. B 104, 663 (2000); S. Chen, J. Am. Chem. Soc. 122, 7420 (2000).

${ }^{17}$ A. K. A. Aliganga, A.-S. Duwez, and S. Mittler, Org. Electron. 7, 337 (2006); A. K. A. Aliganga, I. Lieberwirth, G. Glasser, A.-S. Duwez, and S. Mittler, ibid. 8, 161 (2007).

${ }^{18}$ R. G. Nuzzo and D. L. Allara, J. Am. Chem. Soc. 105, 4481 (1983); C. D. Bain, J. Evall, and G. M. Whitesides, ibid. 111, 321 (1989); R. G. Nuzzo, F. A. Fusco, and D. L. Allara, ibid. 109, 2358 (1987).

${ }^{19}$ A. Ulman, Chem. Rev. (Washington, D.C.) 96, 1533 (1996); A. Ulman, Thin Films: Self-Assembled Monolayers of Thiols (Academic, San Diego, CA, 1998).

${ }^{20}$ J. Käshammer, P. Wohlfart, J. Weib, C. Winter, R. Fischer, and S. Mittler-Neher, Opt. Mater. (Amsterdam, Neth.) 9, 406 (1998); P. Wohlfart, J. Weib, J. Käshammer, C. Winter, V. Scheumann, R. A. Fischer, and S. Mittler-Neher, Thin Solid Films 340, 274 (1999); C. Winter, U. Weckenmann, R. A. Fischer, J. Käshammer, V. Scheumann, and S. Mittler, Chem. Vap. Deposition 6, 199 (2000); R. A. Fischer, U. Weckenmann, C. Winter, J. Käshammer, V. Scheumann, and S. Mittler, J. Phys. IV 11, 1183 (2001).

${ }^{21}$ C. F. Bohren and D. R. Huffman, Absorption and Scattering of Light by Small Particles (Wiley, New York, 1983).

${ }^{22}$ A. Taflove and S. C. Hagness, Computational Electrodynamics: The Finite-Difference Time-Domain Method (Artech House, Boston, 2000).

${ }^{23}$ B. T. Draine and J. J. Goodman, Astrophys. J. 405, 685 (1993).

${ }^{24}$ S. Xu, G. Podoprygorina, V. Böhmer, Z. Ding, P. Rooney, C. Rangan, and S. Mittler, Org. Biomol. Chem. 5, 558 (2007).

${ }^{25}$ D. K. Aswal, S. Lenfant, D. Guerin, J. V. Yakhmi, and D. Vuillaume, Small 1, 725 (2005).
${ }^{26}$ H. Schmidbaur and A. Shiotani, Chem. Ber. 104, 2821 (1971); M. J. Hampden-Smith and T. T. Kodas, Chem. Vap. Deposition 1, 8 (1995).

${ }^{27}$ S. I. Najafi Introduction to Glass Integrated Optics (Artech House, Boston, 1992); R. V. Ramaswamy and R. Srivastavam, J. Lightwave Technol. 6, 984 (1988).

${ }^{28}$ M. J. Adams, An Introduction to Optical Waveguides (Wiley, New York, 1981); R. G. Hunsperger, Integrated Optics: Theory and Technology, 4th ed. (Springer-Verlag, Berlin, 1995); Integrated Optics, 2nd ed., edited by T. Tamir (Springer-Verlag, Heidelberg, 1979); A. W. Snyder and J. D. Love, Optical Waveguide Theory (Chapman and Hall, London, 1983); R. Syms and J. Cozens, Optical Guided Waves and Devices (McGraw-Hill, New York, 1992); D. Marcuse, Theory of Dielectric Optical Waveguides, 2nd ed. (Academic, Boston, 1991).

${ }^{29}$ E. J. Zeman and G. C. Schatz, J. Phys. Chem. 91, 239 (1987).

${ }^{30}$ M. Sukharev and T. Seideman, J. Chem. Phys. 124, 144707 (2006); E. Lidorikis, S. Egusa, and J. D. Joannopoulos, J. Appl. Phys. 101, 54304 (2007).

${ }^{31}$ D. Bedeaux and J. Vlieger, Optical Properties of Surfaces, 2nd ed. (World Scientific, Singapore, 2004).

${ }^{32}$ B. T. Draine and P. J. Flatau, J. Opt. Soc. Am. A 11, 1491 (1994); User Guide for the Discrete Dipole Approximation Code DDSCAT (Version 6.0), http://xxx.lanl.gov/abs/astro-ph/0008151; B. T. Draine and P. J. Flatau, Program DDSCAT.6.0, available at http://www.astro.princeton.edu/ draine/DDSCAT.html.

${ }^{33}$ E. D. Palik, Handbook of Optical Solids (Academic, Orlando, 1985), Vols. 1-3.

${ }^{34}$ T. R. Jensen, M. L. Duval, K. L. Kelly, A. A. Lazarides, G. C. Schatz, and R. P. Van Duyne, J. Phys. Chem. B 103, 9846 (1999); M. D. Malinsky, K. L. Kelly, G. C. Schatz, and R. P. Van Duyne, J. Am. Chem. Soc. 123, 1471 (2001).

${ }^{35}$ R. G. Freeman, K. C. Grabar, K. J. Allison, R. M. Bright, J. A. Davis, A. P. Guthrie, M. B. Hommer, M. A. Jackson, P. C. Smith, D. G. Walter, and M. J. Natan, Science 267, 1629 (1995).

${ }^{36}$ V. V. Klimov and D. V. Guzatov, Phys. Rev. B 75, 024303 (2007). 